\title{
Malaysia's three major ethnic group preferences in creating a Malaysian garden identity
}

\begin{abstract}
Malaysia has a complex multiracial population, predominantly defined by three major ethnic groups: Malay, Chinese and Indian races, with diverse cultural backgrounds. Despite this, the country has a vision to be the most beautiful garden nation, and its National Landscape Policy (NLP) puts emphasis on creating a unique landscape and garden identity. Because of ethnic and cultural differences, there are differences between preferences for developing a garden identity for Malaysia. Hence, this study focused on the visual quality of gardens. Representative images of four well-established gardens of the world including PersianIslamic, English, Japanese and Chinese gardens were presented to the study's respondents through preference photo surveys. Respondents from the three major ethnic groups in Malaysia were asked to rate their preferred garden types, elements and scenes that they would like to see in Malaysian gardens. The results pinpointed expectations of the visual quality of gardens. These highlighted some similarities and differences between the three major ethnic groups in their preferences for the scenes and elements that they sought in gardens. Diverse factors must be considered when creating Malaysian gardens that will be accepted by Malaysians of different ethnic groups. Garden development reveals preferences attached to visual qualities and emphasises cultural differences between garden users.
\end{abstract}

Keyword: Garden development; Preference; Visual quality; Cultural differences. 